\title{
Amphipoda (Crustacea) collected from the Dampier Archipelago, Western Australia
}

\author{
Rachael A. Peart \\ Crustacean Section, The Australian Museum, 6 College Street \\ Sydney, New South Wales 2010, Australia \\ email: rachaelpeart@crustacea.net
}

\begin{abstract}
This paper examines the amphipod fauna of Western Australia. Emphasis is placed on amphipods collected on the 1999 West Australian Museum diving survey of the Dampier Archipelago (DA3/99). The Ampithoidae was identified as one of the most abundant in the survey. The species composition of this family in comparison to other families is discussed. This paper also provides a checklist to the Western Australian amphipod fauna.
\end{abstract}

\section{INTRODUCTION}

This paper reports on the Amphipoda (in particular those of the Ampithoidae) of the Dampier Archipelago, Western Australia. These animals were collected during a survey of the Dampier Archipelago in August and September 1999. This survey was part of the second (DA3/99) of two diving expeditions (DA1/98 and DA3/99) conducted in the Dampier Archipelago as part of the Woodside Energy Ltd./Western Australia Museum partnership.

Amphipods are malacostracan crustaceans of the Peracarida. They are a large group of relatively small crustaceans that live in a variety of habitats. They range in size from $1 \mathrm{~mm}$ to $\sim 300 \mathrm{~mm}$ in length, with a variety of morphologies. Amphipods occur in just about every marine habitat and some freshwater and terrestrial habitats. They have been documented scavenging, burrowing, pelagic and living in and with other organisms. The majority of these lifestyles have been identified for Australian waters.

There are $>8000$ species of amphipod described (Debelius, 1999). These species can by divided into four groups (based on classification prior to Myers and Lowry, in press). These are the Gammaridea, Hyperidea, Ingolfiellidea and the Caprellidae. Each of these groups has been recorded from around Australia. The dominant group in Australia is the Gammaridea with approximately 313 genera in 78 families. The hyperideans have 48 genera in 15 families, the caprellideans 21 genera in five families and the ingolfiellideans one genus in one family (Lowry and Springthorpe, 2001). The majority of these reports have been from eastern or southern Australian waters. This is not due to a lack of diversity in the northern and western areas but a lack of study.
Since 1922 there have only been 11 notable studies which document Western Australian amphipod species (Tattersall, 1922; Barnard, 1972, 1974; Berents, 1983; Barnard and Karaman, 1987; Moore, 1988; Myers, 1988; Jones and Morgan, 1993; Thomas, 1997; Just, 2000, 2002). Some of these studies are based on data from other states and only briefly mention Western Australian species. The most comprehensive study was by Barnard and published in two volumes (Barnard, 1972, 1974). Barnard's study, however, only covered the area from Kalbarri to Albany, with an emphasis on the southwestern corner of the state. A checklist of the species occurring in Western Australian waters is provided (Table 1).

Sampling for amphipods in Western Australian waters has been biased (for example Barnard, 1972, 1974), so that most species have been reported from either shallow water algae or sponges.

\section{MATERIALS AND METHODS}

Collections were made from 35 sites around the islands of the Dampier Archipelago in August and September 1999. The emphasis of this section of the survey was to examine the amphipod fauna on algae and in particular brown seaweed (Phaeophyta) and seagrasses. However, this flora was not present at all the stations and there is, therefore, a bias away from them.

Depending on the site, the amphipods were either collected by hand using SCUBA or from under rocks or scrapings by hand at the intertidal sites. The intertidal samples were mainly taken from scrapings under rocks on sand or mud flats. However, some samples were actually sand scrapings or washings of drift algae. 
All the amphipods collected during the Dampier survey were identified but, due to time constraints and a paucity of species information, not all were taken to the species level (Table 2). Collections are deposited in both the Western Australian Museum (Perth) and the Australian Museum (Sydney).

\section{RESULTS}

Twenty-one families of amphipods were collected from the Dampier Archipelago. It was often difficult to identify these animals to a lower level, as the species could not often be so identified (either through time constraints or they appeared to be new species). The 21 families comprise 68 species in 34 genera (Table 2). The two dominant family groups are the Ampithoidae and the Melitidae. The ampithoids comprise five genera and nine species, totaling 168 specimens. The main melitid group comprises five genera and 13 species, with a total of 109 specimens. Other abundant families include the Ischyroceridae (64 specimens), Deximinidae (54 specimens) and Podoceridae (24 specimens).

Ten families (almost half of those recorded) are new records for Western Australia. This increased level of diversity is also mirrored in the ampithoids at the generic and specific levels. This family has three genera and eight species not previously known from Western Australia. Some species are either new to science or are new records for Western Australia. As there have been no amphipods previously recorded from the Dampier Archipelago, all the species, genera and families are new records.

Nineteen of the 35 samples came from mixed habitats, sampled at between 3-20 m depth by SCUBA. This habitat was either sponge, mixed algae, rocks or coral rubble or any combination of these. At six stations, algae alone were sampled. The remaining 10 stations were intertidal with either algae or rock scrapings. Even though only eight algal stations (both subtidal and intertidal) were sampled, these yielded most species and were dominated by representatives of the Ampithoidae that rarely occurred in the mixed habitats. The dominant group in mixed habitats (both intertidal and subtidal) was the melitid group. Members of this group also occasionally occurred in algal samples.

\section{DISCUSSION}

This study has been invaluable in filling a large gap in the various collections from Western Australia. The Australian amphipod fauna has been mainly documented from eastern and southern Australian coasts. In this study, there are a large number of taxa at the species, genus and family levels which are either new to Western Australia or, at species level, new to science.
In this survey, when amphipod collections were made the emphasis was on collecting representatives of the Ampithoidae. This is because a geographically wider project is currently underway examining the species diversity and biogeography of ampithoid amphipods around the Australian coast (Peart, 2002). As ampithoids are predominantly algal-dwelling amphipods, the bias placed on the survey was that algae were mainly sampled.

Ampithoids were noticeably the most abundant of the algal-dwelling amphipods collected in this survey. This abundance has similar proportions to that recorded recently from Sydney waters (Poore and Lowry, 1997). The Sydney study noted that in algal communities (consisting mainly of the brown alga Sargassum spp) of Port Jackson, the ampithoids were the most abundant and diverse amphipods collected (Poore and Lowry, 1997).

It is curious that in all the previous studies conducted in Western Australia, only three species of Ampithoidae have been recorded. This may be due to biases in sampling protocols or research effort. In a wider study of Australian ampithoids (Peart, 2002), other sites were sampled (Geraldton to Cape Naturaliste) and these have shown that there are at least 15 new species in Western Australian waters. Six of these were identified in this survey of the Dampier Archipelago. The description of these new species will be documented in a later publication.

The other groups of amphipods collected during the survey seem quite comparable, in terms of species numbers, to the groups recorded in other studies. This is shown quite well with the melitid group, which has 13 species recorded from five genera from the Dampier Archipelago (Table 2). Previous studies have recorded eight genera consisting of 21 species (Tattersall, 1922; Barnard, 1972; 1974, Berents, 1983; Barnard and Karaman, 1987; Jones and Morgan, 1993). Species composition also shows some overlap, as with the ampithoids, and there appear to be a number of undescribed species in the collected samples. It is difficult to compare the species richness of amphipods in other families with the ampithoid fauna, as few of them could be identified to the species level. It will take further study and time to determine the exact status of each of these species.

The 1999 survey of the Dampier Archipelago has provided an invaluable resource for further studies of the area and the biogeography of the Australian coastline. Australia is important in determining the distributional range of species throughout the IndoWest Pacific region. Knowing the fauna of this area is important in understanding the processes that are continuously changing and influencing the distribution of species. 


\section{REFERENCES}

Barnard, J.L. (1972). Gammaridean Amphipoda of Australia, Part I. Smithsonian Contributions to Zoology 103: 1-333.

Barnard, J.L. (1974). Gammaridean Amphipoda of Australia, Part II. Smithsonian Contributions to Zoology 139: 1-148.

Barnard, J.L. and Karaman, G.S. (1987). Revisions in classification of gammaridean Amphipoda (Crustacea), Part 3. Proceedings of the Biological Society of Washington 100: 856-875.

Berents, P.B. (1983). The Melitidae of Lizard Island and adjacent reefs, the Great Barrier Reef, Australia (Crustacea: Amphipoda). Records of the Australian Museum 35: 101-143.

Debelius, H. (1999). Crustacea guide of the world, Atlantic Ocean, Indian Ocean, Pacific Ocean. Ikan Unterwasserarchiv, Frankfurt, Germany. 321 pp.

Jones, D.S. and Morgan, G.J. (1993) An annotated checklist of Crustacea from Rottnest Island, Western Australia. In Wells, F.E., Walker, D.I., Kirkman, H. and Lethbridge, R. (eds), Proceedings of the Fifth International Marine Biological Workshop: The Marine Flora and Fauna of Rottnest Island, Western Australia: 135-162. Western Australian Museum, Perth.

Just, J. (2000). Two new species of Exampithoe Barnard, 1925, subgenus Melanesius Ledoyer, 1984, from southern Australia (Crustacea: Amphipoda: Ampithoidae). Records of the Australian Museum 52: 129-136.

Just, J. (2002). Review of Pseudopleonexes Conlan, 1982, with a new species from Australia (Crustacea: Amphipoda: Ampithoidae). Records of the Australian Museum 54: 31-40.
Lowry, J.K. and Springthorpe, R.T. (2001). Amphipoda: Families. Version 1: 2 September 2001. http:// www.crustacea.net

Moore, P.G. (1988). New and little-known marine Amphipoda (Crustacea) from Tasmania and Western Australia. Journal of Natural History 22: 149-174.

Myers, A.A. (1988) The genera Archaeobemlos n.gen., Bemlos Shoemaker, Protolembos Myers and Globosolembos Myers (Amphipoda, Aoridae, Aorinae) from Australia. Records of the Australian Museum 40: 265-332.

Myers, A.A. and Lowry, J.K. (2003). A phylogeny and a new classification of the Corophiidea Leach, 1814 (Amphipoda). Journal of Crustacean Biology 23: 443485.

Peart R.A. (2002). The systematics and phylogeny of the Ampithoidae (Crustacea: Amphipoda), with an emphasis on the Australian fauna. $482 \mathrm{pp}$. Unpublished Ph. D thesis, July 2002, University of New England.

Peart R.A. (2003 in press). A revision of the Cymadusa filosa complex (Crustacea: Amphipoda: Corophioidea: Ampithoidae). Journal of Natural History.

Poore, A.G.B. and Lowry, J.K. (1997). New ampithoid amphipods from Port Jackson, New South Wales, Australia (Crustacea: Amphipoda: Ampithoidae). Invertebrate Taxonomy 11: 897-941.

Tattersall, W.M. (1922). The Percy Sladen Trust expeditions to the Abrolhos Islands (Indian Ocean). Journal of the Linnean Society (Zoology) 35: 1-19.

Thomas, J.D. (1997) Systematics, ecology and phylogeny of the Anamixidae (Crustacea: Amphipoda). Records of the Australian Museum 49: 35-98. 
Table 1 Checklist of Amphipoda recorded from Western Australia. * Described in Peart (2003, in press) ${ }^{* *}$ Recorded in Barnard 1972, 1974

\begin{tabular}{|c|c|}
\hline Taxa & Locality \\
\hline $\begin{array}{l}\text { Family Amaryllidae } \\
\text { Amaryllis carrascoi Lowry and Stoddart, } 2002 \\
\text { Amaryllis dianae Lowry and Stoddart, } 2002 \\
\text { Amaryllis migo Lowry and Stoddart, } 2002 \\
\text { Amaryllis philatelica Lowry and Stoddart, } 2002 \\
\text { Amaryllis quokka Lowry and Stoddart, } 2002 \\
\text { Bamarooka dinjerra Lowry and Stoddart, } 2002 \\
\text { Bamarooka tropicalis Lowry and Stoddart, } 2002\end{array}$ & $\begin{array}{l}\text { King George Sound } \\
\text { Fremantle } \\
\text { Torbay Bay } \\
\text { Foul Bay } \\
\text { Rottnest Island } \\
\text { North West Shelf, Port Hedlar } \\
\text { North West Shelf, Port Hedlar }\end{array}$ \\
\hline $\begin{array}{l}\text { Family Ampithoidae } \\
\text { Cymadusa n. sp. }{ }^{*} \\
\text { Exampithoe compressa Just, } 2000\end{array}$ & $\begin{array}{l}\text { Abrolhos Islands, Cockbum S } \\
\text { Albany, Vancouver Peninsula }\end{array}$ \\
\hline $\begin{array}{l}\text { Family Aoridae } \\
\text { Bemlos ephippium disjuncta Myers, } 1988 \\
\text { Bemlos quadrimanus (Sivaprakasam, 1971) } \\
\text { Bemlos strigilis Myers, } 1988 \\
\text { Protolembos yaranus Myers, } 1988 \\
\text { Xenocheira seurati Chevreux, } 1907\end{array}$ & $\begin{array}{l}\text { Kalbarri } \\
\text { Central west coast } * * \\
\text { Rottnest Island } \\
\text { Kalbarri } \\
\text { Lower west coast } * *\end{array}$ \\
\hline $\begin{array}{l}\text { Family Biancolinidae } \\
\text { Biancolina australis Nicholls, } 1939\end{array}$ & Rottnest Island \\
\hline $\begin{array}{l}\text { Family Caprellidae } \\
\text { Aciconula miranda Mayer, } 1903 \\
\text { Caprella equilibra Say, } 1818 \\
\text { Hemiaegina minuta Mayer, } 1890 \\
\text { Metaprotella haswelliana (Mayer, 1882) } \\
\text { Metaprotella sandalensis Mayer, } 1898 \\
\text { Monoliropus agilis Mayer, } 1903 \\
\text { Noculacia australiensis Guerra-Garcia, } 2002\end{array}$ & $\begin{array}{l}\text { Central west coast } \\
\text { Lower west coast } \\
\text { Lower west coast } \\
\text { Northwest coast } \\
\text { Central and lower west coast } \\
\text { Lower west coast } \\
\text { Northwest and central coast }\end{array}$ \\
\hline
\end{tabular}

\section{Family Ceinidae}

Ceina wannape J.L. Barnard, 1972

Family Cheluridae

Chelura terebrans Philippi, 1837

Family Colomastigidae

Yulumara tricuspis Moore, 1988

Family Corophiidae

Corophium minor Thomson, 1946

Family Cyamidae

Cyamus balaenopterae K.H. Barnard, 1931

Cyamus boopis Lutken, 1870

Cyamus carodontis Margolis, 1954

Cyamus erraticus Roussel de Vauzeme, 1834

Family Cyproideidae

Austropheonoides mulndoe J.L. Barnard, 1972

Cyproidea ornata Haswell, 1879

Narapheonoides mullaya J.L. Barnard, 1972

Unyapheonoides dabber J.L. Barnard, 1972

\section{Family Dexaminidae}

Guernea (Guernea) endota J.L. Barnard, 1972

Guernea (Guernea) melape J.L. Barnard, 1972

Guernea (Guernea) unchalka J.L. Barnard, 1972

Paradexamine churinga J.L. Barnard, 1972

Paradexamine frinsdorfi Sheard, 1938

Paradexamine goomai J.L. Barnard, 1972

Near Albany

Lower west coast **

Seven Mile Beach

Swan River

Central west coast

Central west coast

Southwest corner

Central west coast

Albany

Southwestern coast **

Cape Naturaliste

Cheyne Beach

Cheyne Beach

Cape Naturaliste

Albany

Cockburn Sound

Southwest corner **

Cape Naturaliste 
Paradexamine linga J.L. Barnard, 1972

Paradexamine marlie J.L. Barnard, 1972

Paradexamine narluke J.L. Barnard, 1972

Paradexamine otichi J.L. Barnard, 1972

Paradexamine quarallia J.L. Barnard, 1972

Paradexamine ronggi J.L. Barnard, 1972

Paradexamine thadalee J.L. Barnard, 1972

Paradexamine windarra J.L. Barnard, 1972

Prophlias anomalus Nicholls, 1939

Syndexamine runde J.L. Barnard, 1972

Syndexamine wunda J.L. Barnard, 1972

Family Eophliantidae

Bircenna ignea Nicholls, 1939

Family Eusiridae

Gondogeneia microdeuteropa (Haswell, 1880)

Tethygeneia elanora J.L. Barnard, 1972

Tethygeneia nalgo J.L. Barnard, 1972

Tethygeneia tulkara J.L. Barnard, 1972

Tethygeneia waminda J.L. Barnard, 1972

\section{Family Exoedicerotidae}

Exoedicerotides maculosus (Sheard, 1936)

\section{Family Hyalidae}

Allorchestes compressus Dana, 1852

Hyale crassicornis (Haswell, 1879)

Hyale loorea J.L. Barnard, 1974

Hyale rubra (Thomson, 1879)

Hyale yake J.L. Barnard, 1974

\section{Family Isaeidae}

Gammaropsis (Gammaropsis) atlantica Stebbing, 1888

\section{Family Ischyroceridae}

Ambicholestes (Ambicholestes) cygnatratus Just, 1998

Ambicholestes (Austrolestes) minutus Just, 1998

Australoecetes (Australoecetes) sellicki (Sheard, 1938)

Ericthonius coxacanthus Moore, 1988

Ericthonius pugnax Dana, 1852

\section{Family Leucothoidae}

Anamixis nedcampensis Thomas, 1997

Anamixis ningaloo Thomas, 1997

Leucothoe commensalis Haswell, 1879

Leucothoe gooweera J.L. Barnard, 1974

Leucothoe gracilis (Haswell, 1879)

Paraleucothoe novaehollandiae (Haswell, 1879)

\section{Family Lysianassidae}

Comicostoma karta Lowry and Stoddart, 1983

Lepidepecreum dampieri Lowry and Stoddart, 2002

Parawaldeckia dilkera J.L. Barnard, 1972

Parawaldeckia stebbingi (Thomson, 1893)

Parawaldeckia yamba J.L. Barnard, 1972

Tryphosella orana J.L. Barnard, 1972

Waldeckia chevreuxi Stebbing, 1910

\section{Family Melitidae}

Ceradocus dooliba J.L. Barnard, 1972

Ceradocus rubromaculatus (Stimpson, 1856)

Elasmopus memurte J.L. Barnard, 1974

Elasmopus yunde J.L. Barnard, 1974

Gamarella berringar (J.L. Barnard, 1974)
Cape Naturaliste

Cockburn Sound

Cape Naturaliste

Albany

Albany

Cape Naturaliste

Albany

Albany

Rottnest Island

Southwest coast **

Albany

Nornalup

Southwest corner **

Albany

Albany

Cape Naturaliste

Cape Naturaliste

Lower west coast **

Lower west coast **

Lower west coast **

Cape Naturaliste

Cape Naturaliste

Albany

Abrolhos Islands

Bush Bay, near Carnarvon

North West Shelf

Lower west coast **

Cliff Head

Lower west coast **

Ningaloo Reef

Ningaloo Reef

Lower west coast **

Bluff Point

Lower west coast **

Lower west coast **

Lower west coast **

Between Port Hedland and Dampier Archipelago

Cape Naturaliste

Lower west coast **

Lower west coast **

Albany

Northwest coast **

Lower west coast **

Lower west coast **

Cape Naturaliste

Point Peron

Cottesloe 
Table 1 (cont.)

\begin{tabular}{|c|c|}
\hline Taxa & Locality \\
\hline Hoho carteta (J.L. Barnard, 1972) & Albany \\
\hline Hoho hirtipalma Lowry and Fenwick, 1983 & Lower west coast $* *$ \\
\hline Hoho marilla (J.L. Barnard, 1972) & Lower west coast ** \\
\hline Maera mastersii (Haswell, 1879) & Lower west coast $* *$ \\
\hline Maeracoota sp. & Lower west coast $* *$ \\
\hline Mallacoota diemenesis (Haswell, 1879) & Lower west coast $* *$ \\
\hline Mallacoota subcarinata (Haswell, 1879) & Lower west coast ** \\
\hline Melita matilda J.L. Barnard, 1972 & Swan River \\
\hline Melita oba J.L. Barnard, 1972 & Cape Naturaliste \\
\hline Melita zeylanica kauerti J.L. Barnard, 1972 & Swan River \\
\hline Parelasmopus echo J.L. Barnard, 1972 & Bunbury \\
\hline Parelasmopus ya J.L. Barnard, 1972 & Cockburn Sound \\
\hline Quadrimaera serrata (Schellenberg, 1938) & Abrolhos Islands \\
\hline Quadrimaera viridis (Haswell, 1879) & Lower west coast $* *$ \\
\hline \multicolumn{2}{|l|}{ Family Nihotungidae } \\
\hline Nihotunga iluka J.L. Barnard, 1972 & Cape Naturaliste \\
\hline \multicolumn{2}{|l|}{ Family Phtisicidae } \\
\hline Litiarchus perplexus Mayer, 1912 & Geraldton and Cockburn Sound \\
\hline Pseudoproto fallax Mayer, 1903 & Central west coast \\
\hline \multicolumn{2}{|l|}{ Family Phliantidae } \\
\hline Pereionotus thomsoni Stebbing, 1899 & South-west coast $* *$ \\
\hline Quasimodia barnardi Sheard, 1936 & Lower west coast ${ }^{* *}$ \\
\hline \multicolumn{2}{|l|}{ Family Phoxocephalidae } \\
\hline Birubius batei (Haswell, 1879) & Lower west coast $* *$ \\
\hline Birubius eake Barnard and Drummond, 1978 & Cape Naturaliste \\
\hline Birubius gambodeni Barnard and Drummond, 1978 & Albany \\
\hline Birubius jirrandus Barnard and Drummond, 1978 & Northwest coast $* *$ \\
\hline Birubius nammuldus Barnard and Drummond, 1978 & Barrow Island \\
\hline Brolgus tattersalli (Barnard, 1958) & Abrolhos Islands \\
\hline Ganba pellati Barnard and Drummond, 1978 & Albany \\
\hline Kuritus nacoomus Barnard and Drummond, 1978 & Barrow Island \\
\hline Parharpinia villosa (Haswell, 1879) & Southwest coast ${ }^{* *}$ \\
\hline Uldanamia pillare Barnard and Drummond, 1978 & Lower west coast ** \\
\hline Wildus thambaroo Barnard and Drummond, 1978 & Albany \\
\hline Yan tiendi Barnard and Drummond, 1978 & Albany \\
\hline
\end{tabular}

Family Stegocephalidae

Tetredeion dampieri (Berge and Vader, 2000)

Between Dampier and Port Hedland, North West Shelf

Family Stenothoidae

Ausatelson ule J.L. Barnard, 1972

Chuculba alla J.L. Barnard, 1974

Chuculba warea J.L. Barnard, 1974

Goratelson warroo J.L. Barnard, 1972

Raumahara derroo J.L. Barnard, 1972

Raumahara judithae Moore, 1981

Raumahara noko J.L. Barnard, 1974

Raumahara waroona Krapp-Schickel, 2000

Stenothoe allinga J.L. Barnard, 1974

Stenothoe miersi (Haswell, 1879)

Stenothoe nonedia J.L. Barnard, 1974

Stenothoe quabara J.L. Barnard, 1974

Stenothoe woka J.L. Barnard, 1974

\section{Family Uristidae}

Ichnopus caritus Lowry and Stoddart, 1992

Ichnopus wardi Lowry and Stoddart, 1992

Cape Naturaliste

Albany

Cape Naturaliste

Cape Naturaliste

Cape Naturaliste

Southwest coast ${ }^{* *}$

Southwest coast ${ }^{* *}$

Southwest coast **

Albany

Lower west coast **

Cape Naturaliste

Albany

Albany

King George Sound

North West Shelf

Geographe Bay

Family Urohaustoridae

Urohaustorius vercoi Sheard, 1936 
Table 2 Amphipods recorded from the DA3/99 Dampier Archipelago diving survey. $\mathrm{M}=$ mixed habitat at depth; $\mathrm{A}=$ algal habitat at depth; $\mathrm{I}-\mathrm{A}=$ intertidal algal sample; $\mathrm{I}-\mathrm{M}=$ intertidal mixed habitat. $\mathrm{D} 1-70=$ individual sample numbers at each station. *for details see Station Lists

\begin{tabular}{ll}
\hline Taxa & Station numbers* \\
\hline Family Aoridae & \\
Bemlos sp. & DA3/99/45 (D28b) \\
Globosolembos sp. 1 & DA3/99/56 (D49) \\
Xenocheira sp. & DA3/99/61 (D55) \\
Aorid sp. & DA3/99/59 (D52) \\
& DA3/99/17\#52
\end{tabular}

Family Ampeliscidae

Ampelisca sp. I

DA3/99/41 (D26)

Station type

Abundance

Family Ampithoidae

Ampithoe sp. 1

DA3/99/35 (D8)

DA3/99/45 (D29)

DA3/99/62 (D56)

DA3/99/68 (D61)

Ampithoe cf. kava Myers, 1985

$\mathrm{DA} 3 / 99 / 35$ (D18)

DA3/99/45 (D30, D31, D32, D33)

DA3/99/68 (D61, D63)

Ampithoe sp. 2

DA3/99/44 (D28a)

Ampithoe sp. 3

$\mathrm{DA} 3 / 99 / 45(\mathrm{D} 29)$

$\mathrm{DA} 3 / 99 / 45$ (D30)

$\mathrm{DA} 3 / 99 / 33$ (D36)

DA3/99/17\#52

Cymadusa sp. 4

DA3/99/35 (D11, D12, D13, D14, D18, D19)

DA3/99/37 (D20)

DA3/99/44 (D28a)

DA3/99/45 (D30, D31)

DA3/99/33 (D36)

DA3/99/50 (D44)

DA3/99/56 (D49)

DA3/99/62 (D56)

DA3/99/63 (D57)

DA3/99/68 (D61)

Cymadusa sp. 5

DA3/99/45 (D32, D33)

Exampithoe sp. 6

DA3/99/35 (D14)

DA3/99/42 (D24)

DA3/99/45 (D30, D33)

I-M

$M$

$M$

I-M

?

1

DA3/99/33 (D37)

DA3/99/48 (D42)

Paragrubia sp. 7

DA3/99/35 (D7, D10, D15, D16, D19)

DA3/99/41 (D26)

DA3/99/45 (D29, D32)

DA3/99/33 (D38)

M

1

DA3/99/39 (D22)

A

I-A

I-A

A

A

I-A

A

M

I-A

I-A

A

?

A

I-M

M

I-A

A

M

M

I-A

M

A

I-A

A

I-M

I-A

A

I-M

A

$\mathrm{M}$

I-A

A

M

6
1

1

Family Caprellidae

DA3/99/35 (D13)

DA3/99/45 (D28b)

DA3/99/59 (D52)

DA3/99/44 (D28a)

DA3/99/44 (D28a)

DA3/99/59 (D52)

DA3/99/36 (D1)

DA3/99/35 (D7, D10)

DA3/99/45 (D33)

$\mathrm{DA} 3 / 99 / 47$ (D34, D41)

DA3/99/37 (D20)

DA3/99/44 (D28a)

DA3/99/59 (D52)

DA3/99/59 (D52)

$\begin{array}{ll}\text { A } & 1 \\ \text { I-M } & 2 \\ \text { I-M } & 2 \\ \text { M } & 1 \\ \text { M } & 1 \\ \text { I-M } & 6 \\ \text { M } & 1 \\ \text { A } & 4 \\ \text { I-A } & 2 \\ \text { A } & 7,3 \\ \text { I-M } & 1 \\ \text { M } & 2 \\ \text { I-M } & 1 \\ \text { I-M } & 1\end{array}$

Elasmopus cf. hooheno

Maera sp. 1

Maera cf. hamigera

3
6
$4,5,4,3$
6,1
1
13
$1,1,1,1,1,2$
2
2,8
7
2
1
3
3,3
1
1,1
3
$3,2,3,7,2$
2,7
11


Table 2 (cont.)

\begin{tabular}{|c|c|c|c|}
\hline Taxa & Station numbers* & Station type & Abundance \\
\hline \multirow[t]{2}{*}{ Parelasmopus cf. echo } & $\mathrm{DA} 3 / 99 / 44$ (D28a) & $M$ & 2 \\
\hline & $\mathrm{DA} 3 / 99 / 70(\mathrm{D} 65)$ & $\mathrm{I}-\mathrm{M}$ & 1 \\
\hline \multirow[t]{5}{*}{ Parelasmopus cf. suensis } & $\mathrm{DA} 3 / 99 / 35$ (D9) & A & 1 \\
\hline & $\mathrm{DA} 3 / 99 / 37$ (D20) & $\mathrm{I}-\mathrm{M}$ & 4 \\
\hline & $\mathrm{DA} 3 / 99 / 45$ (D28b) & $\mathrm{I}-\mathrm{M}$ & 13 \\
\hline & $\mathrm{DA} 3 / 99 / 33 ?$ (D36) & A & 1 \\
\hline & $\mathrm{DA} 3 / 99 / 48(\mathrm{D} 42)$ & $\mathrm{I}-\mathrm{M}$ & 1 \\
\hline Parelasmopus cf. ya & $\mathrm{DA} 3 / 99 / 37$ (D20) & $\mathrm{I}-\mathrm{M}$ & 2 \\
\hline \multirow{13}{*}{ Ceradocus group } & $\mathrm{DA} 3 / 99 / 36(\mathrm{D} 2)$ & $\mathrm{M}$ & 5 \\
\hline & $\mathrm{DA} 3 / 99 / 35$ (D12, D17, D18, D19) & $\mathrm{A}$ & $1,4,3,3$ \\
\hline & $\mathrm{DA} 3 / 99 / 42(\mathrm{D} 25)$ & $\mathrm{I}-\mathrm{M}$ & 1 \\
\hline & $\mathrm{DA} 3 / 99 / 45$ (D30) & $\mathrm{I}-\mathrm{A}$ & 1 \\
\hline & $\mathrm{DA} 3 / 99 / 55$ (D48) & $\mathrm{M}$ & 1 \\
\hline & $\mathrm{DA} 3 / 99 / 57$ (D50) & $\mathrm{M}$ & 1 \\
\hline & $\mathrm{DA} 3 / 99 / 58$ (D51) & $\mathrm{M}$ & 1 \\
\hline & $\mathrm{DA} 3 / 99 / 61$ (D55) & $\mathrm{M}$ & 5 \\
\hline & $\mathrm{DA} 3 / 99 / 62$ (D56) & $\mathrm{I}-\mathrm{M}$ & 13 \\
\hline & DA3/99/63 (D57) & $\mathrm{M}$ & 3 \\
\hline & $\mathrm{DA} 3 / 99 / 66$ (D58) & $\mathrm{I}-\mathrm{M}$ & 4 \\
\hline & $\mathrm{DA} 3 / 99 / 68$ (D63) & A & 1 \\
\hline & $\mathrm{DA} 3 / 99 / 17 \# 52$ & $?$ & 2 \\
\hline \multicolumn{4}{|l|}{ Family Colomastigidae } \\
\hline Colomastix sp. & DA3/99/36 (D1) & $\mathrm{M}$ & 1 \\
\hline Colomastigidae sp. & DA3/99/60 (D53) & M & 1 \\
\hline CYPROIDEA & $\mathrm{DA} 3 / 99 / 36$ (D1) & $M$ & 1 \\
\hline \multicolumn{4}{|l|}{ Family Deximinidae } \\
\hline Polycheira sp. & $\mathrm{DA} 3 / 99 / 45$ (D32) & $\mathrm{I}-\mathrm{A}$ & 2 \\
\hline \multirow[t]{5}{*}{ Deximinae sp. } & DA3/99/35 (D8, D9, D10, D19) & A & $1,1,4,2$ \\
\hline & $\mathrm{DA} 3 / 99 / 45$ (D30, D33) & $\mathrm{I}-\mathrm{A}$ & 5,1 \\
\hline & $\mathrm{DA} 3 / 99 / 47$ (D35, D36, D39) & $A$ & $1,1,1$ \\
\hline & DA3/99/68 (D61, D62) & $A$ & 13,21 \\
\hline & $\mathrm{DA} 3 / 99 / 17 \# 52$ & $?$ & 1 \\
\hline \multicolumn{4}{|l|}{ Family Eusiridae } \\
\hline Tethygeneia sp. & DA3/99/35 (D9) & $A$ & 1 \\
\hline \multirow[t]{3}{*}{ Eusiridae sp. } & DA3/99/35 (D7, D11, D12) & A & $1,1,1$ \\
\hline & DA3/99/45 (D30, D31) & $\mathrm{I}-\mathrm{A}$ & 2,1 \\
\hline & DA3/99/47 (D34, D37, D40) & A & $4,1,3$ \\
\hline \multirow[t]{2}{*}{ Family Hyalidae } & $\mathrm{DA} 3 / 99 / 45$ (D30, D32) & $\mathrm{I}-\mathrm{A}$ & 2,6 \\
\hline & DA3/99/59 (D52) & $\mathrm{I}-\mathrm{M}$ & 10 \\
\hline Family Iciliidae? & $\mathrm{DA} 3 / 99 / 56(\mathrm{D} 49)$ & M & 1 \\
\hline IPHIMEDIODEA & $\mathrm{DA} 3 / 99 / 68$ (D61) & A & 1 \\
\hline \multirow[t]{5}{*}{ Family Isaeidae } & $\mathrm{DA} 3 / 99 / 35$ (D14) & $A$ & 1 \\
\hline & $\mathrm{DA} 3 / 99 / 42$ (D26) & $M$ & 8 \\
\hline & $\mathrm{DA} 3 / 99 / 45$ (D33) & $\mathrm{I}-\mathrm{A}$ & 1 \\
\hline & $\mathrm{DA} 3 / 99 / 47$ (D41) & A & 3 \\
\hline & $\mathrm{DA} 3 / 99 / 55$ (D48) & $M$ & 1 \\
\hline \multicolumn{4}{|l|}{ Family Ischyroceridae } \\
\hline \multirow[t]{4}{*}{ Cerapus sp. } & $\begin{array}{l}\text { DA3/99/35 (D6, D7, D8, D9, D11, D12, } \\
\text { D13, D14, D15, D16, D17, D18) }\end{array}$ & $\mathrm{A}$ & $\begin{array}{l}2,5,2,6,5,3,1,1 \\
1,4,2,2\end{array}$ \\
\hline & $\mathrm{DA} 3 / 99 / 45$ (D31, D33) & $\mathrm{I}-\mathrm{A}$ & 1,5 \\
\hline & $\mathrm{DA} 3 / 99 / 47$ (D40) & A & 1 \\
\hline & DA3/99/68 (D61, D63) & $A$ & 3,6 \\
\hline
\end{tabular}


Taxa

Ischyroceridae $\mathrm{sp}$.

Leucothoe cf. goowera

Leucothoidae sp.

Family Liljeborgidae

LYSSIANASSOIDEA

Family Oedicerotidae

Family Phliantidae

Family Phoxocephalidae

Family Podoceridae
Station numbers*

DA3/99/36 (D1)

DA3 $/ 99 / 35$ (D18)

DA3/99/47 (D34, D35, D38)

DA3/99/56 (D49)

DA3/99/56 (D49)

DA3/99/36 (D1)

DA3/99/55 (D48)

DA3/99/44 (D28a)

DA3/99/61 (D55)

DA3/99/63 (D57) :

DA3/99/41 (D26)

DA3/99/68 (D63)

DA3/99/59 (D52)

DA3/99/35 (D10, D17)

DA3/99/45 (D33)

DA3/99/47 (D39)

DA3/99/44 (D28a)

DA3/99/35 (D15)

DA3/99/45 (D29)

DA3/99/47 (D38)

DA3/99/68 (D61, D62)
Station type

Abundance

M

A

1

A

$2,2,3$

$\mathrm{M}$

5

$\begin{array}{ll}\mathrm{M} & 2 \\ \mathrm{M} & 1\end{array}$

$\mathrm{M}$

M 2

M i

M 2

M 1

A 1

I-M 15

A 1,1

I-A 1

A 1

M 2

A 2

I-A 2

A

A $\quad 15,1$

